\title{
Front Matter: Volume 10538
}

, "Front Matter: Volume 10538," Proc. SPIE 10538, Optical Interconnects XVIII, 1053801 (27 April 2018); doi: 10.1117/12.2322634

SPIE. Event: SPIE OPTO, 2018, San Francisco, California, United States 


\section{PROCEEDINGS OF SPIE}

\section{Optical Interconnects XVIII}

Henning Schröder

Ray T. Chen

Editors

29-31 January 2018

San Francisco, California, United States

Sponsored and Published by

SPIE 
The papers in this volume were part of the technical conference cited on the cover and title page. Papers were selected and subject to review by the editors and conference program committee. Some conference presentations may not be available for publication. Additional papers and presentation recordings may be available online in the SPIE Digital Library at SPIEDigitallibrary.org.

The papers reflect the work and thoughts of the authors and are published herein as submitted. The publisher is not responsible for the validity of the information or for any outcomes resulting from reliance thereon.

Please use the following format to cite material from these proceedings:

Author(s), "Title of Paper," in Optical Interconnects XVIII, edited by Henning Schröder, Ray T. Chen, Proceedings of SPIE Vol. 10538 (SPIE, Bellingham, WA, 2018) Seven-digit Article CID Number.

ISSN: 0277-786X

ISSN: 1996-756X (electronic)

ISBN: 9781510615618

ISBN: 9781510615625 (electronic)

Published by

SPIE

P.O. Box 10, Bellingham, Washington 98227-0010 USA

Telephone +1 3606763290 (Pacific Time) · Fax +1 3606471445

SPIE.org

Copyright (c) 2018, Society of Photo-Optical Instrumentation Engineers.

Copying of material in this book for internal or personal use, or for the internal or personal use of specific clients, beyond the fair use provisions granted by the U.S. Copyright Law is authorized by SPIE subject to payment of copying fees. The Transactional Reporting Service base fee for this volume is $\$ 18.00$ per article (or portion thereof), which should be paid directly to the Copyright Clearance Center (CCC), 222 Rosewood Drive, Danvers, MA 01923. Payment may also be made electronically through CCC Online at copyright.com. Other copying for republication, resale, advertising or promotion, or any form of systematic or multiple reproduction of any material in this book is prohibited except with permission in writing from the publisher. The CCC fee code is 0277 $786 \mathrm{X} / 18 / \$ 18.00$.

Printed in the United States of America.

Publication of record for individual papers is online in the SPIE Digital Library.

\section{SPIE. DIGITAL}

Paper Numbering: Proceedings of SPIE follow an e-First publication model. A unique citation identifier (CID) number is assigned to each article at the time of publication. Utilization of CIDs allows articles to be fully citable as soon as they are published online, and connects the same identifier to all online and print versions of the publication. SPIE uses a seven-digit CID article numbering system structured as follows:

- The first five digits correspond to the SPIE volume number.

- The last two digits indicate publication order within the volume using a Base 36 numbering system employing both numerals and letters. These two-number sets start with 00, 01, 02, 03, 04, $05,06,07,08,09,0 A, 0 B \ldots$ OZ, followed by 10-1Z, 20-2Z, etc. The CID Number appears on each page of the manuscript. 


\title{
Contents
}

\author{
$\checkmark$ Authors \\ vii Conference Committee
}

\section{SESSION 1 OPTICAL INTERCONNECT SYSTEMS I}

1053804 Universal test system for system embedded optical interconnect [10538-3]

1053805 A low-latency high-port count optical switch with optical delay line buffering for disaggregated data centers (Invited Paper) [10538-4]

SESSION 2 OPTICAL INTERCONNECT SYSTEMS II

1053807 Optical datacenter network employing slotted (TDMA) operation for dynamic resource allocation (Invited Paper) [10538-6]

1053808 A low-latency optical switch architecture using integrated $\mu \mathrm{m}$ SOI-based contention resolution and switching [10538-7]

1053809 On-off keying transmitter design for navigation by visible light communication [10538-8]

10538 OA Visible light communication technology for fine grained indoor localization [10538-9]

SESSION 3 HYBRID INTEGRATED OPTICAL LINK MODULES

$10538 \mathrm{OB}$ Towards co-packaging of photonics and microelectronics in existing manufacturing facilities (Invited Paper) [10538-10]

10538 OD Demonstration of glass based photonic interposer for mid-board optical engines and electrical-optical circuit board (EOCB) integration strategy [10538-12]

10538 OF Organic-inorganic hybrid material SUNCONNECT® for photonic integrated circuit (Invited Paper) [10538-14]

10538 OG Silicon photonic IC embedded optical PCB for high-speed interconnect application [10538-15] 
$10538 \mathrm{OL} \quad$ Broadband infrared light emitting waveguides based on UV curable PbS quantum dot composites [10538-20]

10538 ON Flexible multimode polymer waveguides for high-speed short reach communication links [10538-22]

\section{SESSION 6 NANOPHOTONICS FOR OPTICAL INTERCONNECTS}

10538 OP A silicon reconfigurable optical processor based on a self-coupled optical waveguide [10538-24]

\section{SESSION 7 FIBER OPTICS AND MICRO-OPTICS INTEGRATION}

10538 OS Low profile fiber connector for co-packaged optics (Invited Paper) [10538-28]

10538 OU Automated and model-based assembly of an anamorphic telescope [10538-31]

10538 OV Reduced diameter fibers for high-density optical interconnects (Invited Paper) [10538-32]

SESSION 8 OPTICAL INTERCONNECT DEVICES AND MODULATORS

10538 OY All silicon approach to modulation and detection at $\boldsymbol{\lambda}=\mathbf{2} \boldsymbol{\mu m}$ (Invited Paper) [10538-35]

SESSION 9 PICS FOR OPTICAL INTERCONNECTS

1053812 Laser printed glass planar lightwave circuits with integrated fiber alignment structures [10538-39]

SESSION 10 HYBRID SILICON OPTICAL SYSTEMS AND DEVICES I: JOINT SESSION WITH CONFERENCES 10537 AND 10538

1053816 Optical interconnects based on VCSELs and low-loss silicon photonics (Invited Paper) [10538-43]

POSTER SESSION

10538 1D Polarization division multiplexing for optical data communications [10538-50]

iv 


\section{Authors}

Numbers in the index correspond to the last two digits of the seven-digit citation identifier (CID) article numbering system used in Proceedings of SPIE. The first five digits reflect the volume number. Base 36 numbering is employed for the last two digits and indicates the order of articles within the volume. Numbers start with 00, 01, 02, 03, 04, 05, 06, 07, 08, 09, OA, OB...0Z, followed by 10-12, 20-2Z, etc.

Aalto, Timo, 08,16

Ackert, Jason J., OY

Avramopoulos, H., 07

Aziz, M., 07

Baig, Sarfaraz, OL

Bakopoulos, P., 07

Bamiedakis, N., ON

Barwicz, Tymon, OB

Bickham, Scott R., OV

Bougie, Jerome, OB

Boyer, Nicolas, OB

Brusberg, Lars, OS

Butler, Douglas L., OS

Cao, Wei, OY

Capitani, M., 07

Chalk, Julie A., OV

Chamberlain, Roger D., 1D

Chen, Jianping, $\mathrm{OP}$

Cherchi, Matteo, 08, 16

Childers, Darrell, OB

Christodoulopoulos, K., 07

Chu, D., ON

Clark, Jeffrey S., OS

Costa, J., 09

Cyr, Elaine, $\mathrm{OB}$

DeJong, Michael, OS

Desmet, A., 12

Dirks, Sebastian, OU

Drouin, Alain, $\mathrm{OB}$

Engelmann, Sebastian, $O B$

Fantoni, A., OA

Fortier, Paul, OB

Gruev, Viktor, 1D

Guo Xin, Tina, OY

Hagan, David, OY

Harjanne, Mikko, 08, 16

Holters, Martin, OU

Hurley, William C., OV

Immonen, M., 04

Ivanovich, Darko, 1D

Janta-Polczynski, Alexander, OB

Jiang, Guomin, OL

Kallega, Rakshitha, OG

Kamlapurkar, Swetha, OB

Karppinen, Mikko, 16

Kashino, Tsubasa, OF

Kim, Sung Jin, OL

Knights, Andrew P., OY

Krawczyk, Jakub, OV
Kumar, Abhai, OG

Kyriakos, A., 07

Landi, G., 07

Langlois, Richard, OB

Lichoulas, Ted W., OB

Littlejohns, Callum G., OY

Loosen, Peter, OU

Louro, P., 09, 0A

LU, Liangjun, OP

Malacarne, Antonio, 16

Mashanovich, Goran Z., OY

Missinne, J., 12

Moralis-Pegios, M., 05, 08

Mourgias-Alexandris, G., 05, 08

Nambiar, Siddharth, $O G$

Nawata, Hideyuki, OF

Nedeljkovic, Milos, OY

Neitz, M., 04, OD

Neumeyr, Christian, 16

Olila, Jyrki, 16

Oshima, Juro, OF

Paap, Mark T., OV

Paik, Young-hun, $\mathrm{OL}$

Patronas, I., 07

Penty, R. V., ON

Pitwon, R., 04

Pleros, N., 05, 08

Powell, Samuel B., 1D

Przemyska, Magdalena, OV

Qiu, Haodong, OY

Radosavljevic, A., 12

Ranganath, Praveen, OG

Reed, Graham T., OY

Reisis, D., 07

Ripumaree, Radawan, OV

Rouifed, Mohamed Saïd, OY

Schneider-Ramelow, M., OD

Schröder, H., 04, OD

Selvaraja, Shankar Kumar, OG

Shen, Kai, OL

Shen, Lin, OP

Shi, F., ON

Sitomaniemi, Aila, 16

Soler Penades, Jordi, OY

Spatharakis, C., 07

Stollenwerk, Jochen, OU

Sutton, Clifford G., OS

Taira, Yoichi, OB

Takenobu, Shotaro, OB 
Terzenidis, N., 05, 08

Thomson, David J., OY

Tokas, K., 07

Van Steenberge, G., 12

Van Thourhout, D., 12

Varvarigos, E., 07

Vieira, M. A., 09, 0A

Vieira, M., 09, 0A

Vieira, P., OA

Vyrsokinos, K., 05, 08

Wang, Hong, $\mathrm{OY}$

Wang, K., 04

Wang, Michael R., OL

Wang, Wanjun, OY

White, I. H., ON

Zahavi, E., 07

Zhang, Zecen, OY

Zhou, Linjie, OP

Proc. of SPIE Vol. 10538 1053801-6

Downloaded From: https://www.spiedigitallibrary.org/conference-proceedings-of-spie on 26 Apr 2023 Terms of Use: https://www.spiedigitallibrary.org/terms-of-use 


\title{
Conference Committee
}

\author{
Symposium Chairs
}

Connie J. Chang-Hasnain, University of California, Berkeley (United States)

Graham T. Reed, Optoelectronics Research Centre, University of Southampton (United Kingdom)

Symposium Co-Chairs

Jean-Emmanuel Broquin, IMEP-LAHC (France)

Shibin Jiang, AdValue Photonics, Inc. (United States)

Program Track Chairs

Yakov Sidorin, Quarles \& Brady LLP (United States)

Benjamin Dingel, Nasfine Photonics, Inc. (United States)

\section{Conference Chairs}

Henning Schröder, Fraunhofer-Institut für Zuverlässigkeit und Mikrointegration (Germany)

Ray T. Chen, The University of Texas at Austin (United States)

Conference Program Committee

Darrell Childers, US Conec Ltd. (United States)

Alan F. Evans, Corning Incorporated (United States)

Alexei L. Glebov, OptiGrate Corporation (United States)

Ruth Houbertz, Multiphoton Optics GmbH (Germany)

Marika P. Immonen, TTM Technologies, Inc. (Finland)

Takaaki Ishigure, Keio University (Japan)

Wei Jiang, Nanjing University (China)

Mikko Karppinen, VTT Technical Research Center of Finland (Finland)

Christian Koos, Karlsruher Institut für Technologie (Germany)

Tobias Lamprecht, vario-optics ag (Switzerland)

Christopher T. Middlebrook, Michigan Technological University (United States)

Bert-Jan Offrein, IBM Research - Zürich (Switzerland)

Hyo-Hoon Park, KAIST (Korea, Republic of)

Nikos Pleros, Aristotle University of Thessaloniki (Greece)

Richard C. Pitwon, Seagate Systems (UK) Ltd. (United Kingdom)

Brandon W. Swatowski, Dow Corning Corporation (United States)

David J. Thomson, University of Southampton (United Kingdom) 
Alan X. Wang, Oregon State University (United States)

Michael R. Wang, University of Miami (United States)

Ian H. White, University of Cambridge (United Kingdom)

Chris Q. Wu, Corning Incorporated (United States)

Xiaochuan Xu, Omega Optics, Inc. (United States)

\section{Session Chairs}

$1 \quad$ Optical Interconnect Systems I

Henning Schröder, Fraunhofer-Institut für Zuverlässigkeit und Mikrointegration (Germany)

2 Optical Interconnect Systems II

Ray T. Chen, The University of Texas at Austin (United States)

3 Hybrid Integrated Optical Link Modules

Chris Q. Wu, Corning Incorporated (United States)

4 Electrical-Optical PCB Technologies

Richard C. Pitwon, Seagate Systems (UK) Ltd. (United Kingdom)

5 Novel Optical Waveguide and Interconnect Technologies

Takaaki Ishigure, Keio University (Japan)

6 Nanophotonics for Optical Interconnects

Ruth Houbertz, Multiphoton Optics GmbH (Germany)

7 Fiber Optics and Micro-Optics Integration

Darrell Childers, US Conec Ltd. (United States)

8 Optical Interconnect Devices and Modulators

Christian Koos, Karlsruher Institut für Technologie (Germany)

9 PICs for Optical Interconnects

Christopher T. Middlebrook, Michigan Technological University (United States)

10 Hybrid Silicon Optical Systems and Devices I: Joint Session with Conferences 10537 and 10538

Alan X. Wang, Oregon State University (United States)

11 Hybrid Silicon Optical Systems and Devices II: Joint Session with Conferences 10537 and 10538

Andrew P. Knights, McMaster University (Canada) 